\title{
Diagnostic Value of Model-Based Iterative Reconstruction Combined with a Metal Artifact Reduction Algorithm during CT of the Oral Cavity
}

\author{
(D) Y. Kubo, (D) K. Ito, (D) M. Sone, (D). Nagasawa, (D). Onishi, (D). Umakoshi, (D)T. Hasegawa, (D). Akimoto, and (D). Kusumoto
}

\begin{abstract}
BACKGROUND AND PURPOSE: Metal artifacts reduce the quality of CT images and increase the difficulty of interpretation. This study compared the ability of model-based iterative reconstruction and hybrid iterative reconstruction to improve CT image quality in patients with metallic dental artifacts when both techniques were combined with a metal artifact reduction algorithm.

MATERIALS AND METHODS: This retrospective clinical study included 40 patients (men, 31; women, 9; mean age, $62.9 \pm 12.3$ years) with oral and oropharyngeal cancer who had metallic dental fillings or implants and underwent contrast-enhanced ultra-high-resolution CT of the neck. Axial CT images were reconstructed using hybrid iterative reconstruction and model-based iterative reconstruction, and the metal artifact reduction algorithm was applied to all images. Finally, hybrid iterative reconstruction + metal artifact reduction algorithms and model-based iterative reconstruction + metal artifact reduction algorithm data were obtained. In the quantitative analysis, SDs were measured in ROls over the apex of the tongue (metal artifacts) and nuchal muscle (no metal artifacts) and were used to calculate the metal artifact indexes. In a qualitative analysis, 3 radiologists blinded to the patients' conditions assessed the image-quality scores of metal artifact reduction and structural depictions.
\end{abstract}

RESULTS: Hybrid iterative reconstruction + metal artifact reduction algorithms and model-based iterative reconstruction + metal artifact reduction algorithms yielded significantly different metal artifact indexes of 82.2 and 73.6, respectively $(95 \% \mathrm{Cl}, 2.6-14.7$; $P<.01)$. The latter algorithms resulted in significant reduction in metal artifacts and significantly improved structural depictions $(P<.01)$.

CONCLUSIONS: Model-based iterative reconstruction + metal artifact reduction algorithms significantly reduced the artifacts and improved the image quality of structural depictions on neck CT images.

ABBREVIATIONS: $I R$ = iterative reconstruction; hybrid-IR+MAR = combination of hybrid iterative reconstruction and metal artifact reduction algorithms; $M A R=$ metal artifact reduction algorithms; MBIR = model-based iterative reconstruction; MBIR+MAR = combination of model-based iterative reconstruction and metal artifact reduction algorithms; U-HRCT = ultra-high-resolution CT

M any patients have metallic dental fillings or implants, which are highly attenuating objects that often cause metal artifacts on CT and thus limit the diagnostic value of these data by reducing the image quality. ${ }^{1}$ On $\mathrm{CT}$, these artifacts comprise

Received April 13, 2020; accepted after revision July 7.

From the Department of Diagnostic Radiology (Y.K., K.I., M.S., H.N., Y.O., N.U., T.H., M.K.), National Cancer Center Hospital, Tokyo, Japan; Department of Cancer Medicine (Y.K., T.A.), Jikei University Graduate School of Medicine, Tokyo, Japan; and Division of Radiation Oncology and Particle Therapy (T.A.), National Cancer Center Hospital East, Kashiwa, Japan.

The study was supported by a grant from Canon Medical Systems.

Paper previously presented, in part, at: Annual Meeting of the European Society of Head and Neck Radiology, October 3-5, 2019; Palermo, Italy.

Please address correspondence to Yuko Kubo, MD, 5-1-1 Tsukiji, Chuo-ku, Tokyo, 104-0045 Japan; e-mail: yukkubo@ncc.go.jp

- Indicates open access to non-subscribers at www.ajnr.org

http://dx.doi.org/10.3174/ajnr.A6767 areas of low or high density that appear as streaks or radial foci with variable levels of brightness. ${ }^{2}$ Metal artifacts can also cause areas of whiteout, where CT numbers around the metallic object exceed the maximum CT number range, or blackout, where no image data are visible. Consequently, several artifact reduction methods have been developed to improve the quality of images produced by modern CT systems.

Iterative reconstruction (IR) was initially developed to preserve the quality of a CT image while reducing the level of noise. ${ }^{3}$ Although iterative reconstruction methods have been available since 1970, the limited computational power available at that time meant that this option was not feasible in clinical settings due to the overly long duration of image reconstruction. Therefore, a simpler approach (filtered back-projection) was used because it allowed faster processing and greater feasibility in clinical settings. Iterative reconstruction did not reappear until 2009. ${ }^{4}$ 
Unlike conventional filtered back-projection, which is based on simpler mathematic assumptions of the tomographic imaging system, ${ }^{5}$ IR is used to generate a set of synthesized projections by accurately modeling the data-collection process in CT. Hybrid iterative reconstruction (hybrid-IR) approaches apply some noisereduction techniques to sinograms and image spaces. Hybrid-IR provides much better image quality and potentially enables reductions in radiation doses. ${ }^{6-8}$ Recently, the evolution of hybrid-IR led to model-based iterative reconstruction (MBIR). This fully iterative algorithm minimizes the difference between the measured original sinogram and the sinogram reproduced by forward projection and uses a more complex system of prediction models that account for scanner hardware parameters, the conebeam trajectory, and the photoelectric trajectory. ${ }^{9,10}$ Compared with earlier hybrid-IR techniques, MBIR provides superior image resolution at lower radiation doses. ${ }^{11-17}$ MBIR has also been reported to constitute a useful approach for metal artifact reduction. ${ }^{18-21}$

Another approach for the reduction of metal artifacts involves the use of dedicated metal artifact reduction algorithms (MAR). With time, researchers have described the remarkable ability of MAR to enhance the visualization of various target lesions by reducing metallic artifacts. ${ }^{18,22-29}$ Therefore, the intended effect of MAR has been established, and the use of CT with MAR comprises the current clinical standard.

Recent advances have made possible the combination of MBIR and MAR. This study aimed to clarify differences in the degree of metal artifact reduction and depiction of oral cavity structures between hybrid-IR and MBIR when both techniques were combined with MAR and to evaluate the ability of the latter to improve image quality and diagnostic value.

\section{MATERIALS AND METHODS}

This retrospective single-institution study was approved by the institutional review board of the National Cancer Center Hospital, Tokyo, Japan. The institutional review board waived the requirement for written informed consent from patients due to the design of the study and the use of anonymized patient records and data.

\section{Patient Characteristics}

The inclusion criteria were the diagnosis of oral or oropharyngeal carcinoma with tumors of $>2 \mathrm{~cm}$ (longest diameter) and evaluation by contrast-enhanced ultra-high-resolution CT (U-HRCT) at our hospital between October 2017 and August 2018. The exclusion criteria were as follows: 1) a history of an oral cavity operation, 2) a lack of dental fillings or implants, and 3) a lack of raw $\mathrm{CT}$ data required for reconstruction.

For all patients, the age, sex, tumor location, histopathologic cancer type, and the number and sites of dental fillings or implants were determined. In cases involving the placement of dental fillings on multiple adjacent teeth, such as dental bridges, the number of originally treated teeth was counted. The size of the dental fillings or implants was not considered because these were $\leq 12 \mathrm{~mm}$ per tooth and thus equal to or less than the size of the original tooth.

\section{CT}

All images were acquired on a U-HRCT scanner (Aquilion Precision, Canon Medical Systems) in super-high-resolution mode (1792 channels/detector row, $0.25 \times 160$ rows; matrix size, 1024). The scanning parameters were a rotation time of $0.5 \mathrm{sec}-$ onds, pitch factor of 0.569 , scanning FOV of $24 \mathrm{~cm}$, and voltage of $120 \mathrm{kV}$. Automatic tube current modulation was used in all examinations, resulting in a mean tube current of $272.4 \pm 38.2 \mathrm{~mA}$, mean CT dose index of $14.5 \pm 1.3 \mathrm{mGy}$, and mean dose-length product of $511.9 \pm 57.4 \mathrm{mGy}$. A body weight-adapted volume of iodinated contrast medium (iopromide, Ultravist, $370 \mathrm{mg} / \mathrm{mL}$; Bayer HealthCare) was administered intravenously at a flow rate of $1.6-2.0 \mathrm{~mL} / \mathrm{s}$ for 50 seconds. The scan was acquired at a delay of 80 seconds after the commencement of contrast injection.

Axial images of the neck were reconstructed from helical scan data using the following algorithms: hybrid-IR (Adaptive Iterative Dose Reduction 3D [AIDR3D]; Canon Medical Systems) and MBIR (Forward projected model-based Iterative Reconstruction SoluTion [FIRST]; Canon Medical Systems); then, MAR (Single Energy Metal Artifact Reduction [SEMAR]; Canon Medical Systems) was applied to all images. Finally, hybrid-IR+MAR and MBIR+MAR were performed. A total of 80 image sets (40 patients) were obtained by reconstruction. AIDR3D reconstructions of areas of soft tissue were created using the standard reconstruction FC13 kernel. FIRST was reconstructed using a BODY kernel equivalent to a standard reconstruction kernel. The following parameters were identical across the reconstruction algorithms: $\mathrm{z}$-axis range, frontal sinus to cricoid cartilage; section thickness, $3.0 \mathrm{~mm}$; section interval, $3.0 \mathrm{~mm}$; and reconstruction FOV, $24 \mathrm{~cm}$.

\section{Quantitative Image Analysis}

The quantitative image analyses were performed by a board-certified radiologist (Y.K., with 14 years of experience). Circular ROIs were placed over the tongue apex in an area containing metal artifacts $\left(\sim 400 \mathrm{~mm}^{2}\right)$ and over the nuchal muscle at the level of the hyoid bone in an area without metal artifacts $\left(\sim 100 \mathrm{~mm}^{2}\right)$. The copy-paste function was used to ensure that the sizes and locations of the ROIs were identical across both reconstruction algorithms applied to data from a single patient. The mean CT attenuation value (Hounsfield units) and SD of all ROIs were measured twice, and the average value of the 2 corresponding measures was applied.

The SD of the ROI is a widely used measure of noise on radiographic images. ${ }^{30}$ When used in biologic tissue, the SD represents a combination of the tissue heterogeneity and noise (which may also be caused by metal). In a section containing a metal object, the $\mathrm{SD}$ is affected by both the image noise and the metal artifacts. Calculating the index between the SDs of the muscle (no metal artifacts) and tongue (metal artifacts) reduces the influence of tissue heterogeneity, though this assumes a consistent level of tissue heterogeneity. According to previous studies, ${ }^{31,32}$ the artifact index (AI) used to quantify the severity of metal artifacts is defined as

$$
\mathrm{AI}=\left[\left(\mathrm{SD}_{\mathrm{TONGUE}}\right)^{2}-\left(\mathrm{SD}_{\mathrm{MUSCLE}}\right)^{2}\right]^{1 / 2},
$$

where $\mathrm{SD}_{\mathrm{TONGUE}}$ and $\mathrm{SD}_{\mathrm{MUSCl}}$ denote the $\mathrm{SD}$ of the tongue apex and nuchal muscle, respectively.

The number of reconstructed CT slices was counted, and the time required per CT section reconstructed by hybrid-IR + MAR or MBIR+MAR was measured.

AJNR Am J Neuroradiol 41:2132-38 Nov 2020 www.ajnr.org 


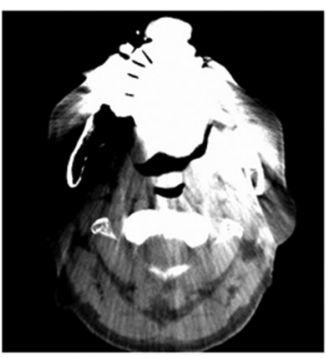

Score 1

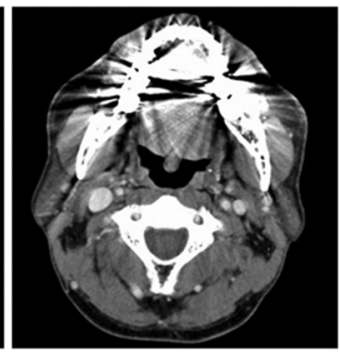

Score 2

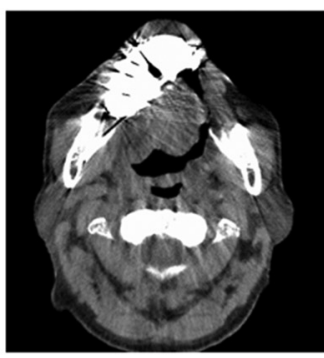

Score 3

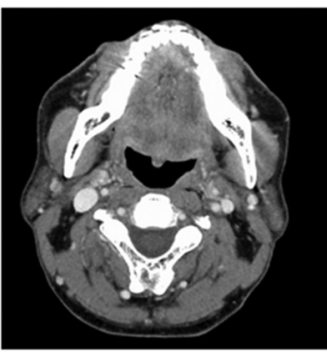

Score 4

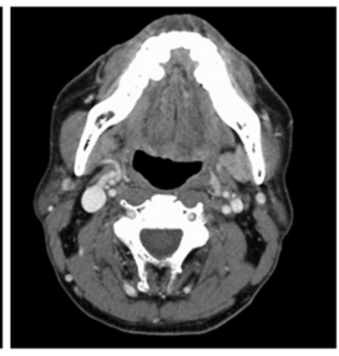

Score 5

FIG 1. Representative CT images of the degree of metal artifact reduction. The image quality of metal artifact reduction was scored using the following 5-point scale: 1, severe metal artifacts in a large area; 2, severe metals artifact in a small area; 3, moderate metal artifacts; 4, minimal metal artifacts; and 5, no metal artifacts.

Assessed for eligibility $(n=71)$

Patient had oral or oropharyngeal carcinoma with tumors $>2 \mathrm{~cm}$ and underwent contrast-enhanced U-HRCT

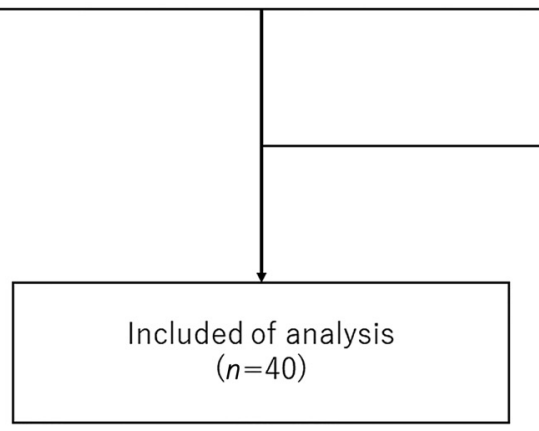

FIG 2. Flow diagram of the inclusion of potentially eligible patients.

\section{Qualitative Analyses}

Three board-certified radiologists (N.U., T.H., and Y.O., with 3, 8, and 9 years of experience, respectively) performed a qualitative analysis of the CT images in consensus. All readers were blinded to the patient demographics and CT parameters and given standardized instructions and training on image sets from 5 patients not included in this study. The readers used the same monitor (RadiForce RX440; EIZO), and the study images were presented in random order on a preset soft-tissue window (window width and level, 325 and $60 \mathrm{HU}$, respectively).

The readers evaluated the image-quality scores of metal artifact reduction and the depictions of representative structures (apex and base of the tongue, parapharyngeal space, superior portion of the internal jugular chain, parotid gland) on a 5-point scale (5, no artifacts/ excellent visualization; 4, minimal artifacts/good visualization; 3 , moderate metal artifacts/acceptable visualization for diagnosis; 2 , severe metal artifacts in a small area/poor visualization; and 1, severe metal artifacts in a large area/no visualization), as shown in Fig 1. Finally, the readers assigned a diagnostic tumor stage according to the criteria of the American Joint Committee on Cancer, Cancer Staging Manual, 8th edition ${ }^{33}$ and examined the correlation between the qualitative metal artifact reduction score and the number of dental fillings or implants in each patient.

\section{Statistical Analysis}

The SPSS Statistics 25 software package (IBM) was used to perform the statistical analyses. The paired Student $t$ test was used to assess quantitative differences among the CT images reconstructed using hybrid-IR+MAR and MBIR+MAR. The Wilcoxon signed rank test was used to evaluate qualitative differences in image-quality scores between the 2 reconstruction algorithms. In the qualitative analysis, we calculated each of the weighted $\kappa$ statistics for all combinations of the 2 readers to assess the degree of agreement among the 3 readers (ie, interobserver agreement). $\kappa$ statistics of $0.81-$ $1.00,0.61-0.80,0.41-0.60,0.21-0.40$, or $0.00-0.20$ were interpreted as excellent, substantial, moderate, fair, or poor agreement, respectively. ${ }^{34}$ The relationship between the number of dental fillings or implants and the metal artifact reduction imagequality score in the qualitative analysis was analyzed by calculating the Spearman rank correlation coefficient. Specifically, using Excel 2016 (Microsoft), we created an approximation curve by plotting the mean scores of the 3 readers that corresponded to the number of dental fillings or implants on a scatter diagram. The level of statistical significance was set at $P<.05$.

\section{RESULTS}

\section{Patient Characteristics}

Figure 2 presents the flow diagram used to determine the inclusion of potentially eligible patients. Overall, 40 patients were included. Table 1 presents the additional demographic and clinical characteristics of the study population. The number of metallic dental fillings or implants per patient ranged from 1 to 23 (median, 11). These fillings or implants were bilateral, right-sided, and left-sided in 37,2 , and 1 patient, respectively.

\section{Quantitative Analyses}

Table 2 presents the findings from quantitative image analyses. At the apex of the tongue and nuchal muscle, the mean CT 
Table 1: Characteristics of the study population

\begin{tabular}{lc}
\multicolumn{1}{c}{ Variable } & Value \\
\hline Age (mean) (yr) & $62.9 \pm 12.3$ \\
Sex (male/female) & $31: 9$ \\
Tumor location & 11 \\
Oral tongue & 11 \\
Tonsil & 6 \\
Floor of mouth & 4 \\
Palate & 4 \\
Base of tongue & 3 \\
Gingiva & 1 \\
Buccal mucosa & \\
Histopathologic type & 32 \\
Squamous cell carcinoma & 3 \\
Malignant lymphoma & 2 \\
Clear cell carcinoma & 1 \\
Adenocarcinoma & 1 \\
Adenosquamous cell carcinoma & 1 \\
Mucoepidermoid carcinoma & \\
\hline
\end{tabular}

Table 2: Quantitative evaluation of $\mathrm{CT}$ attenuation (HU) of the apex of the tongue and nuchal muscle

\begin{tabular}{ccccc}
\hline & $\begin{array}{c}\text { Hybrid- } \\
\text { IR+MAR } \\
\text { (Mean) }\end{array}$ & $\begin{array}{c}\text { MBIR+MAR } \\
\text { (Mean) }\end{array}$ & $95 \% \mathrm{Cl}$ & \begin{tabular}{c} 
D \\
\cline { 3 - 5 } Value $^{\mathrm{a}}$
\end{tabular} \\
\hline $\begin{array}{c}\text { Mean of apex of } \\
\text { the tongue } \\
\text { (HU) }\end{array}$ & $103.7 \pm 52.5$ & $91.9 \pm 50.6$ & $8.2-15.4$ & $<.01^{\mathrm{b}}$ \\
$\begin{array}{c}\text { Mean of nuchal } \\
\text { muscle (HU) }\end{array}$ & $64.9 \pm 6.7$ & $64.0 \pm 6.7$ & $0.1-1.5$ & $.025^{\mathrm{b}}$ \\
$\begin{array}{c}\text { SD of apex of } \\
\text { the tongue } \\
\text { (HU) }\end{array}$ & $82.9 \pm 56.0$ & $74.1 \pm 64.1$ & $2.7-14.8$ & $<.01^{\mathrm{b}}$ \\
$\begin{array}{c}\text { SD of nuchal } \\
\text { muscle (HU) }\end{array}$ & $8.9 \pm 1.6$ & $7.5 \pm 2.0$ & $1.1-1.7$ & $<.01^{\mathrm{b}}$ \\
\hline
\end{tabular}

${ }^{\text {a }}$ Paired $t$ test.

${ }^{\mathrm{b}}$ Significant difference.

attenuation value and SD from MBIR+MAR were significantly lower than those from hybrid-IR+MAR $(P<.01)$. At the apex of the tongue, hybrid-IR+MAR and MBIR + MAR yielded mean metal artifact index values of 82.2 (95\% CI, 64.2-100.2) and 73.6 (95\% CI, 53.0-94.1), respectively, and these values differed significantly between the 2 algorithms (95\% CI, 2.6-14.7; $P<.01$ ). Notably, MBIR + MAR led to a reduction of $11 \%$ in the metal artifact index relative to hybrid-IR+MAR (Fig 3).

The median number of CT slices reconstructed was 102 (range, 83-118). The reconstruction times by hybrid-IR+MAR and MBIR+MAR were 2.1 and 37.6 seconds per section, respectively. In other words, MBIR+MAR required a reconstruction time $>17$ times longer than that of hybrid-IR+MAR.

\section{Qualitative Analyses}

The weighted $\kappa$ statistic revealed excellent interobserver agreement among the 3 readers ( $\kappa$ range, 0.88-0.98). A comparison of the metal artifact reduction image-quality scores revealed that MBIR+MAR yielded significant reductions in metal artifacts compared with hybrid-IR+MAR $(P<.01)$. Figure 4 demonstrates that MBIR+MAR improved the delineation of the oral structures and oral cavity cancers by significantly reducing metal artifacts relative to those produced by hybrid-IR + MAR.

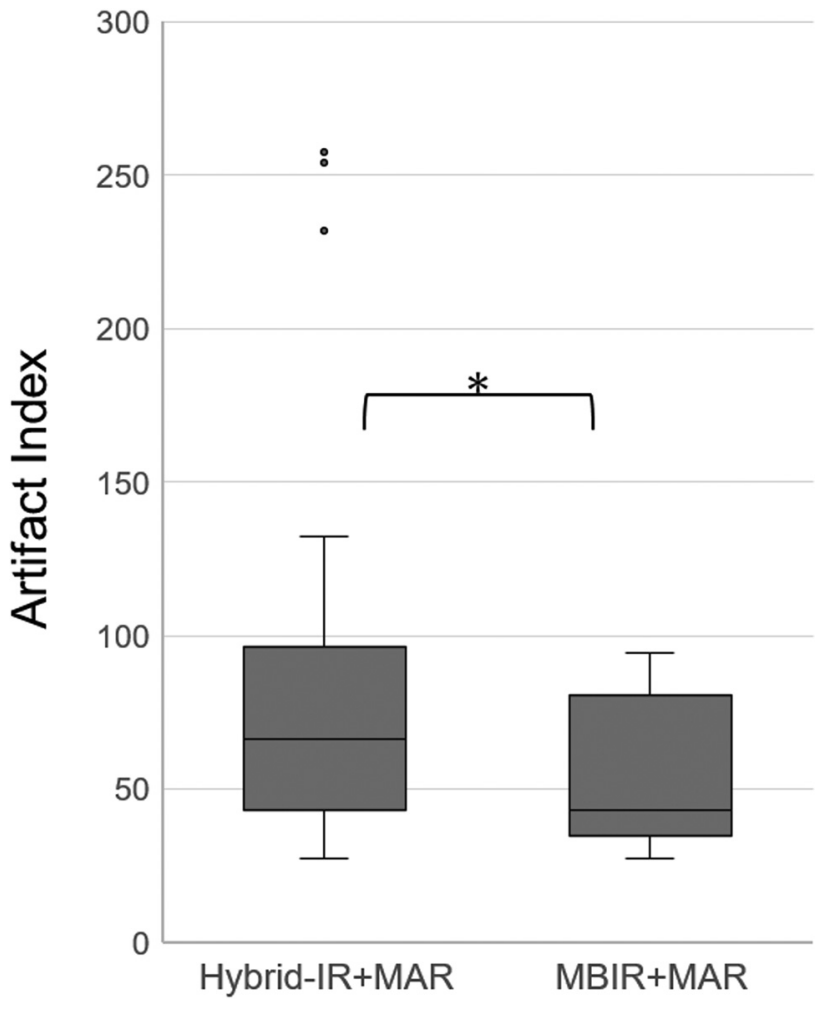

FIG 3. Boxplot comparison of the artifact index values calculated using the 2 reconstruction algorithms: hybrid-IR+MAR and MBIR+MAR. The asterisk indicates a significant difference $(P<.01)$.

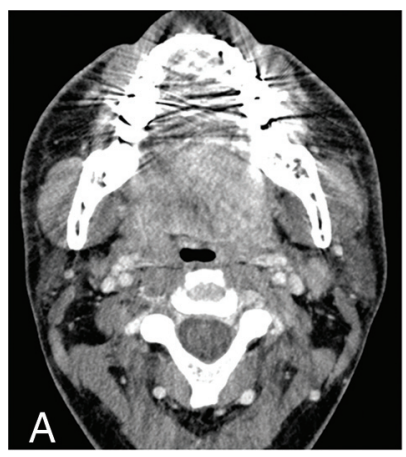

Hybrid-IR+MAR

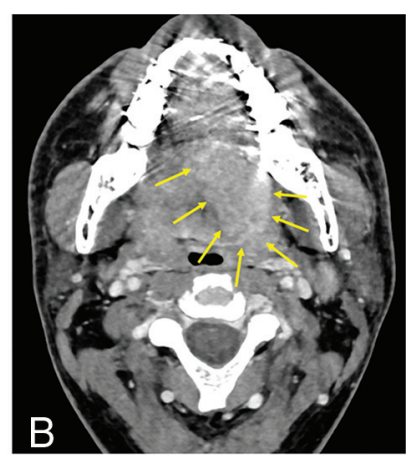

MBIR+MAR
FIG 4. Axial CT images of a 32-year-old man with left-tongue cancer. Reconstruction was performed using hybrid-IR+MAR $(A)$ and MBIR+MAR (B). As shown, MBIR+MAR provided superior reduction of dental artifacts and a better depiction of the tumor (arrows).

Table 3 presents the image-quality scores of the depicted neck structures. All representative structures except the apex of the tongue had a mean score of $\geq 3$, which was within the identifiable visual level; the apex of the tongue only had a score of $\geq 3$ using MBIR+MAR. Comparisons revealed significant differences in the average scores obtained using the 2 reconstruction algorithms. MBIR+MAR yielded higher average scores for all representative structural depictions compared with hybrid-IR+MAR.

Finally, the approximate power curve indicated a negative relationship between the number of dental fillings or implants and the metal artifact reduction image-quality score, regardless of 
Table 3: Qualitative evaluation of image-quality scores of representative structures

\begin{tabular}{lccc}
\hline \multicolumn{1}{c}{ Representative Structures } & $\begin{array}{c}\text { Hybrid-IR+MAR } \\
\text { (Mean) }\end{array}$ & $\begin{array}{c}\text { MBIR+MAR } \\
\text { (Mean) }\end{array}$ & $\begin{array}{c}\boldsymbol{P} \\
\text { Value }^{\mathrm{a}}\end{array}$ \\
\hline Apex of the tongue & $2.78 \pm 0.72$ & $3.02 \pm 0.75$ & $<.01^{\mathrm{b}}$ \\
Base of the tongue & $4.11 \pm 0.29$ & $4.27 \pm 0.41$ & $<.01^{\mathrm{b}}$ \\
Parapharyngeal space & $4.58 \pm 0.33$ & $4.68 \pm 0.27$ & $<.01^{\mathrm{b}}$ \\
Superior portion of internal jugular vein & $4.77 \pm 0.24$ & $4.88 \pm 0.18$ & $<.01^{\mathrm{b}}$ \\
Parotid gland & $4.50 \pm 0.40$ & $4.67 \pm 0.37$ & $<.01^{\mathrm{b}}$ \\
\hline
\end{tabular}

${ }^{a}$ Wilcoxon signed rank test.

bSignificant difference.

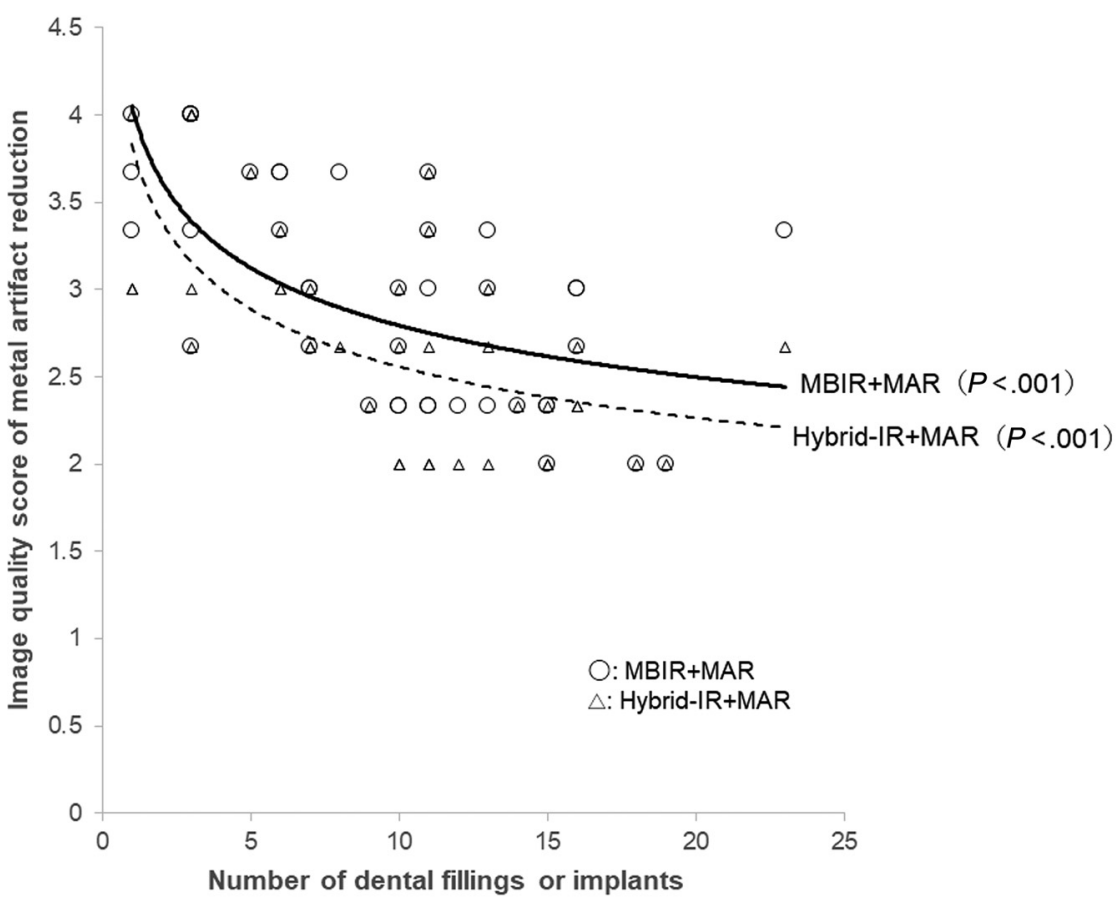

FIG 5. Analysis of the correlation between the image-quality score of artifact reduction and the number of dental fillings or implants between the 2 reconstruction algorithms: hybrid-IR+MAR and MBIR+MAR.

the reconstruction algorithm used. Higher scores were achieved with MBIR+MAR than with hybrid-IR+MAR (Fig 5). The cancer tumor stage diagnoses did not differ significantly with respect to the type of reconstruction algorithm.

\section{DISCUSSION}

This study aimed to compare the CT image quality achieved using a combination of MBIR and MAR versus that achieved with a combination of hybrid-IR and MAR in a sample of patients with oral or oropharyngeal cancer. Our quantitative analysis demonstrated that MBIR+MAR significantly reduced both the mean CT attenuation at the apex of the tongue and the metal artifact index compared with hybrid-IR+MAR. Also, our qualitative analysis demonstrated that MBIR+MAR yielded a more significant reduction of metal artifacts in the oral cavity than hybrid-IR+MAR. In other words, objective and subjective in vivo evaluations revealed significantly better artifact reduction and image quality with $\mathrm{MBIR}+\mathrm{MAR}$ relative to hybrid-IR+MAR.
CT is considered a first-line diagnostic technique for oral cancer because of its broad availability, capacity for wholebody tumor staging, and good overall detection of sensitivity and specificity. ${ }^{35}$ However, it is essential to achieve an excellent image quality with minimal artifacts despite the presence of metal dental fillings or implants. Prior studies indicated a superior reduction of dental artifacts caused by dental hardware or diverse maxillofacial metal implants when several MAR algorithms from major vendors were used, compared with standard reconstruction. ${ }^{24-29}$ However, MAR algorithms may introduce new artifacts into the image. These new artifacts can appear as defects or blurring around metal hardware in the bone window. ${ }^{26,27}$ Recently, several clinical studies reported that the combination of spectral detector CT (or dualenergy CT) with virtual monoenergetic images and MAR provided optimal artifact reduction and improved diagnostic imaging assessments in patients with dental implants and bridges or metallic dental prostheses. ${ }^{36,37}$

As noted previously, the MBIR algorithm is a revolutionary reconstruction technology that uses various models and repeats the subtraction of original raw data after forward projection to yield a reconstructed image that differs minimally from the raw data. In addition to reducing image noise, only the MBIR algorithm reduces streak and beam-hardening artifacts, respectively. ${ }^{19}$ In a previous study, MBIR similarly reduced metal artifacts in the pelvis, spine, oral cavity, and extremities, which was to a greater extent than filtered backprojection or hybrid-IR. ${ }^{18-20}$ Previous reports indicated a better reduction of metal artifacts when hybrid-IR+MAR was used, compared with MBIR without MAR. ${ }^{21,38}$ These results suggest that both MBIR and MAR can effectively reduce metal artifacts. Therefore, this in vivo study investigated the usefulness of MBIR+MAR for reducing metal artifacts in the oral cavity. We found that compared with hybrid-IR+MAR, MBIR+MAR provided better representations of normal neck structures and reduced metal artifacts. We attribute the improved image quality achieved with MBIR+MAR to the incorporation of MBIR settings such as the focus size, detector size, and voxel size, which would improve the spatial resolution. In previous analyses, Wellenberg et $\mathrm{al}^{39}$ and Neroladaki et $\mathrm{al}^{40}$ reported that MBIR+MAR significantly reduced orthopedic metal artifacts on pelvic CT images produced by other vendors., Although the results of those studies were consistent with our findings, the 
methods differed from ours in that either phantoms or small numbers of large orthopedic metal implants were used.

A previous phantom study observed that the metal artifacts increased and the CT image accuracy decreased as the number of metal implants in the oral cavity increased. ${ }^{41}$ From in our vivo study, we also concluded that the metal artifacts worsened as the number of dental fillings increased. MBIR+MAR was relatively less affected by an increased number of dental fillings or implants. Overall, our results demonstrate the specific clinical impact of the combination of MBIR and MAR, even though the metallic artifacts could not be removed completely. Specifically, this in vivo study revealed a reduction of metal artifacts on CT images of the neck region when using $\mathrm{MBIR}+\mathrm{MAR}$.

Our study had some limitations: First, U-HRCT, which first became commercially available in 2017, uses smaller detector elements equivalent to a quarter of the area of the elements in a conventional CT detector. In a previous report, U-HRCT with improved spatial resolution was shown to reduce the artifacts associated with calcified lesions in coronary arteries. ${ }^{42}$ Theoretically, U-HRCT with small detector elements would affect metal artifact reduction by improving the spatial resolution. In our study, however, all patients were scanned using UHRCT; therefore, we could not evaluate the specific reduction of metal artifacts by U-HRCT. Second, in addition to dental fillings or implants, metal plates and screws used in postoperative applications are a major cause of image degradation and a main obstacle to the follow-up of local recurrence in patients with oral cancer. However, these devices were set as an exclusion criterion in this study. Third, the patient population was relatively small and derived from a single institution, and the study design was retrospective. Further studies with much larger samples are needed to reject null hypotheses with clinically negligible differences. ${ }^{43}$ Fourth, the inclusion of only patients with oropharyngeal and oral cancers with solid tumors of $>2 \mathrm{~cm}$ might have led to selection bias. Ideally, we would have included early cancers because these would be most susceptible to metal artifacts. Finally, although MBIR+MAR has some advantages over hybrid-IR+MAR, as demonstrated in our study, it is also disadvantaged by significant computational requirements. Consequently, a longer reconstruction period is required. Future studies should aim to reduce the reconstitution time and increase the practical application of MBIR+MAR.

\section{CONCLUSIONS}

The combination of MBIR and MAR enabled the significant reduction of metal artifacts during oral cavity CT. Moreover, this reconstruction algorithm improved the depiction of structures in the neck with a minimal dependence on the number of dental fillings or implants.

Disclosures: Yuko Kubo-RELATED: Grant: Canon Medical Systems.* Masahiko Kusumoto-RELATED: Grant: Canon Medical Systems*; UNRELATED: Payment for Lectures Including Service on Speakers Bureaus: Ono Pharmaceutical, AstraZeneca KK, MSD KK, Comments: I received honoraria for lecture fees from Ono Pharmaceutical, AstraZeneca KK, and MSD KK. Miyuki Sone-RELATED: Grant: Canon Medical Systems.* *Money paid to the institution.

\section{REFERENCES}

1. Goerres GW, Hany TF, Kamel E, et al. Head and neck imaging with PET and PET/CT: artefacts from dental metallic implants. Eur J Nucl Med Mol Imaging 2002;29:367-70 CrossRef Medline

2. Barrett JF, Keat N. Artifacts in CT: recognition and avoidance. Radiographics 2004;24:1679-91 CrossRef Medline

3. Ravishankar S, Ye JC, Fessler JA. Image reconstruction: from sparsity to data-adaptive methods and machine learning. Proc IEEE Inst Electr Electron Eng 2020;108:86-109 CrossRef Medline

4. Willemink MJ, Noel PB. The evolution of image reconstruction for CT: from filtered back projection to artificial intelligence. Eur Radiol 2019;29:2185-95 CrossRef Medline

5. Herman GT. Fundamentals of computerized tomography: image reconstruction from projections. Springer-Verlag: London, 2009 CrossRef

6. Singh S, Kalra MK, Hsieh J, et al. Abdominal CT: comparison of adaptive statistical iterative and filtered back projection reconstruction techniques. Radiology 2010;257:373-83 CrossRef Medline

7. Gervaise A, Osemont B, Lecocq S, et al. CT image quality improvement using adaptive iterative dose reduction with wide-volume acquisition on 320-detector CT. Eur Radiol 2012;22:295-301 CrossRef Medline

8. Martinsen ACT, Sæther $\mathrm{HK}, \mathrm{Hol} \mathrm{PK}$, et al. Iterative reconstruction reduces abdominal CT dose. Eur J Radiol 2012;81:1483-87 CrossRef Medline

9. Yu Z, Thibault JB, Bouman CA, et al. Fast model-based X-ray CT reconstruction using spatially nonhomogeneous ICD optimization. IEEE Trans Image Process 2011;20:161-75 CrossRef Medline

10. Fleischmann D, Boas FE. Computed tomograph: old ideas and new technology. Eur Radiol 2011;21:510-17 CrossRef Medline

11. Chang W, Lee JM, Lee K, et al. Assessment of a model-based, iterative reconstruction algorithm (MBIR) regarding image quality and dose reduction in liver computed tomography. Invest Radiol 2013;48:598-606 CrossRef Medline

12. Goodenberger MH, Wagner-Bartak NA, Gupta S, et al. Computed tomography image quality evaluation of a new iterative reconstruction algorithm in the abdomen (adaptive statistical iterative reconstruction-V): a comparison with model-based iterative reconstruction, adaptive statistical iterative reconstruction, and filtered back projection reconstructions. J Comput Assist Tomogr 2018;42:184-90 CrossRef Medline

13. Noda Y, Goshima S, Koyasu H, et al. Renovascular CT: comparison between adaptive statistical iterative reconstruction and model-based iterative reconstruction. Clin Radiol 2017;72:901.e913-19 CrossRef Medline

14. Smith EA, Dillman JR, Goodsitt MM, et al. Model-based iterative reconstruction: effect on patient radiation dose and image quality in pediatric body CT. Radiology 2014;270:526-34 CrossRef Medline

15. Taguchi N, Oda S, Imuta M, et al. Model-based iterative reconstruction in low-radiation-dose computed tomography colonography: preoperative assessment in patients with colorectal cancer. Acad Radiol 2018;25:415-22 CrossRef Medline

16. Yasaka K, Katsura M, Akahane M, et al. Model-based iterative reconstruction and adaptive statistical iterative reconstruction: dose-reduced CT for detecting pancreatic calcification. Acta Radiol Open 2016;5:205846011662834 CrossRef Medline

17. Yuki H, Oda S, Utsunomiya D, et al. Clinical impact of model-based type iterative reconstruction with fast reconstruction time on image quality of low-dose screening chest CT. Acta Radiol 2016;57:295-302 CrossRef Medline

18. De Crop A, Casselman J, Van Hoof T, et al. Analysis of metal artifact reduction tools for dental hardware in CT scans of the oral cavity: $\mathrm{kVp}$, iterative reconstruction, dual-energy CT, metal artifact reduction software-does it make a difference? Neuroradiology 2015;57:841-49 CrossRef Medline

19. Boudabbous S, Arditi D, Paulin E, et al. Model-based iterative reconstruction (MBIR) for the reduction of metal artifacts on CT. AJR Am J Roentgenol 2015;205:380-85 CrossRef Medline 
20. Kuya K, Shinohara Y, Kato A, et al. Reduction of metal artifacts due to dental hardware in computed tomography angiography: assessment of the utility of model-based iterative reconstruction. Neuroradiology 2017;59:231-35 CrossRef Medline

21. Yasaka K, Kamiya K, Irie R, et al. Metal artefact reduction for patients with metallic dental fillings in helical neck computed tomography: comparison of adaptive iterative dose reduction $3 \mathrm{D}$ (AIDR 3D), forward-projected model-based iterative reconstruction solution (FIRST) and AIDR 3D with single-energy metal artefact reduction (SEMAR). Dentomaxillofac Radiol 2016;45:20160114 CrossRef Medline

22. Li H, Noel C, Chen H, et al. Clinical evaluation of a commercial orthopedic metal artifact reduction tool for CT simulations in radiation therapy. Med Phys 2012;39:7507-17 CrossRef Medline

23. Brook OR, Gourtsoyianni S, Brook A, et al. Spectral CT with metal artifacts reduction software for improvement of tumor visibility in the vicinity of gold fiducial markers. Radiology 2012;263:696-705 CrossRef Medline

24. Lell MM, Meyer E, Kuefner MA, et al. Normalized metal artifact reduction in head and neck computed tomography. Invest Radiol 2012;47:415-21 CrossRef Medline

25. Kidoh M, Nakaura T, Nakamura S, et al. Reduction of dental metallic artefacts in CT: value of a newly developed algorithm for metal artefact reduction (O-MAR). Clin Radiol 2014;69:e11-16 CrossRef Medline

26. Diehn FE, Michalak GJ, DeLone DR, et al. CT dental artifact: comparison of an iterative metal artifact reduction technique with weighted filtered back-projection. Acta Radiol Open 2017;6:205846011774327 CrossRef Medline

27. Hakim A, Slotboom J, Lieger O, et al. Clinical evaluation of the iterative metal artefact reduction algorithm for post-operative CT examination after maxillofacial surgery. Dentomaxillofac Radiol 2017;46:20160355 CrossRef Medline

28. Weiß J, Schabel C, Bongers M, et al. Impact of iterative metal artifact reduction on diagnostic image quality in patients with dental hardware. Acta Radiol 2017;58:279-85 CrossRef Medline

29. Niehues SM, Vahldiek JL, Tröltzsch D, et al. Impact of single-energy metal artifact reduction on CT image quality in patients with dental hardware. Comput Biol Med 2018;103:161-66 CrossRef Medline

30. Lubner MG, Pickhardt PJ, Tang J, et al. Reduced image noise at lowdose multidetector CT of the abdomen with prior image constrained compressed sensing algorithm. Radiology 2011;260:248-56 CrossRef Medline

31. Lin XZ, Miao F, Li JY, et al. High-definition CT Gemstone spectral imaging of the brain: initial results of selecting optimal monochromatic image for beam-hardening artifacts and image noise reduction. J Comput Assist Tomogr 2011;35:294-97 CrossRef
32. Wang Y, Qian B, Li B, et al. Metal artifacts reduction using monochromatic images from spectral CT: evaluation of pedicle screws in patients with scoliosis. Eur J Radiol 2013;82:e360-66 CrossRef Medline

33. Lydiatt WM, Patel SG, O'Sullivan B, et al. Head and neck cancers: major changes in the American Joint Committee on Cancer Eighth Edition Cancer Staging Manual. CA Cancer J Clin 2017;67:122-37 CrossRef Medline

34. Svanholm H, Starklint H, Gundersen HJ, et al. Reproducibility of histomorphologic diagnoses with special reference to the kappa statistic. APMIS 1989;97:689-98 CrossRef Medline

35. Blatt $S$, Ziebart T, Krüger $M$, et al. Diagnosing oral squamous cell carcinoma: How much imaging do we really need? A review of the current literature. J Craniomaxillofac Surg 2016;44:538-49 CrossRef Medline

36. Cha J, Kim HJ, Kim ST, et al. Dual-energy CT with virtual monochromatic images and metal artifact reduction software for reducing metallic dental artifacts. Acta Radiol 2017;58:1312-19 CrossRef Medline

37. Laukamp KR, Zopfs D, Lennartz S, et al. Metal artifacts in patients with large dental implants and bridges: combination of metal artifact reduction algorithms and virtual monoenergetic images provides an approach to handle even strongest artifacts. Eur Radiol 2019;29:4228-38 CrossRef Medline

38. Toso S, Laurent M, Lozeron ED, et al. Iterative algorithms for metal artifact reduction in children with orthopedic prostheses: preliminary results. Pediatr Radiol 2018;48:1884-90 CrossRef Medline

39. Wellenberg RH, Boomsma MF, van Osch JA, et al. Computed tomography imaging of a hip prosthesis using iterative model-based reconstruction and orthopaedic metal artefact reduction: a quantitative analysis. J Comput Assist Tomogr 2016;40:971-78 CrossRef Medline

40. Neroladaki A, Martin SP, Bagetakos I, et al. Metallic artifact reduction by evaluation of the additional value of iterative reconstruction algorithms in hip prosthesis computed tomography imaging Medicine 2019;98:e14341 CrossRef Medline

41. Tsuchida $\mathrm{Y}$, Takahashi $\mathrm{H}$, Watanabe $\mathrm{H}$, et al. Effects of number of metal restorations and mandibular position during computed tomography imaging on accuracy of maxillofacial models. J Prosthodont Res 2019;63:239-44 CrossRef Medline

42. Motoyama S, Ito H, Sarai M, et al. Ultra-high-resolution computed tomography angiography for assessment of coronary artery stenosis. Circ J 2018;82:1844-51 CrossRef Medline

43. Faber J, Fonseca LM. How sample size influences research outcomes. Dental Press J Orthod 2014;19:27-29 CrossRef Medline 